\title{
Carving Out Parameter Space in Type-II Two Higgs Doublets Model
}

\author{
Benjamín Grinstein, ${ }^{a}$ Patipan Uttayarat ${ }^{b, c}$ \\ ${ }^{a}$ Department of Physics, University of California at San Diego, La Jolla, CA 92093 \\ ${ }^{b}$ Department of Physics, University of Cincinnati, Cincinnati, OH 45220 \\ ${ }^{c}$ Department of Physics, Srinakharinwirot University, Wattana, Bangkok 10110 Thailand \\ E-mail: bgrinstein@ucsd.edu, uttayapn@ucmail.uc.edu
}

\begin{abstract}
We analyze the Type-II two Higgs doublets model in light of the newly discovered Higgs-like particle with mass $125 \mathrm{GeV}$. The observed $125 \mathrm{GeV}$ particle is identified with the light CP-even Higgs boson in the two Higgs doublets model. We study the parameter space of the model consistent with the Higgs data, branching ratio of $\bar{B} \rightarrow X_{s} \gamma$ as well as precision electroweak measurements. We also incorporate theoretical constraints - perturbativity of the couplings and vacuum stability, in our study. We find that only a small parameter space of the model remains viable. The phenomenology of the heavy Higgs bosons in the surviving parameter space is studied.
\end{abstract}




\section{Contents}

1 Introduction $\quad 1$

2 The Model $\quad 3$

2.1 Scalar Sector 4

3 Fit to the Higgs Data $\quad 5$

3.1 Production Cross-sections and Branching Ratios 5

$\begin{array}{lll}3.2 & \text { Global Fit } & 7\end{array}$

4 Bounds on Parameter Space $\quad 7$

4.1 Theoretical Constraints 7

4.2 Experimental Bounds 8

4.3 Viable Parameter Space 9

5 Phenomenology Of The Other Higgs Bosons 10

5.1 Phenomenology of the CP-even $H \quad 11$

5.2 Phenomenology of the CP-odd Boson 13

5.3 Phenomenology of the Charged Boson $H^{ \pm} \quad 15$

6 Conclusions and Discussions 16

A Loop-induced Decays of Neutral Scalar Bosons $\quad 19$

$\begin{array}{ll}\text { B Beta-functions } & 21\end{array}$

\section{Introduction}

Recently the ATLAS and CMS collaborations announced the discovery of a Higgs-like particle with a mass $M_{h} \simeq 125 \mathrm{GeV}[1,2]$. Evidence of this new particle has also been reported by the CDF and D collaborations [3]. However, it is far from certain that this newly discovered particle is the standard model (SM) Higgs boson responsible for electroweak symmetry breaking. The couplings of this Higgs-like particle deviate (although not statistically significantly) from SM expectations [4-7]. Even with the 
updated measurements [8-15], the deviations still remain. Thus it is possible that this Higgs-like particle is a hint of new physics beyond the standard model.

A particularly well motivated class of new physics is the two Higgs doublet model in which electroweak symmetry is broken by two elementary scalar fields. Famously, the minimal supersymmetric standard model (MSSM) contains two higgs doublets to account for masses of all quarks and leptons, and the parameters are constrained by supersymmetry. However, in this work we consider a more generic two Higgs doublet model. For a recent review of a general two Higgs doublet model see Ref. [16] and references therein. In particular we will focus on the CP conserving type II two Higgs doublet model (2HDM-II) in which one scalar field couples only to the up-type quarks and the other couples to the down-type quarks and leptons.

There exists a large literature on the properties of the neutral scalar boson couplings of the two Higgs doublets model in light of the Higgs data; see for example Ref. [17-23]. In this work, in addition to performing a global fit to the currently available Higgs data, we also consider the viable parameter space of the model and study the phenomenology of the other Higgs bosons. ${ }^{1}$ Specifically, we assume that there is no other states except those of the 2HDM-II up to some cutoff scale, $\Lambda$. Thus if there is no viable parameter space for a specific value of the cutoff, we can conclude that, if there is new physics beyond the standard model, the 2HDM-II cannot be the only new physics below that cutoff scale.

From theoretical view point, the model has to allow for an electroweak symmetry breaking vacuum. We also impose a constraint on perturbativity of the coupling constants of the model. What we mean by perturbativity will be made clear in section 4.1. We insist that perturbativity must be satisfied at all energy scales up to the cutoff scale [24]. This is different from Ref [20] which seems to impose perturbativity only at the electroweak scale.

Existing experimental data also constrain possible new physics. The absence of large flavor changing neutral interactions places a strong bound on the mass of the charged Higgs boson. Similarly, the success of the standard model in describing precision electroweak measurements constrains possible new physics states. In this work, we will utilize both experimental and theoretical constraints in determining a viable parameter space for the 2HDM-II.

The paper is organized as follows. In section 2 we briefly describe the 2HDMII and we set our conventions and notations. Then we perform a global fit to the Higgs data in section 3. In section 4 we subject the 2HDM-II to both theoretical and

\footnotetext{
${ }^{1}$ Ref. [17] also studied the viable parameter space of the 2HDM-II but didn't discuss the phenomenology of the other Higgs bosons.
} 
experimental constraints to determine the viable parameter space. We briefly discuss the phenomenology of the heavy CP-even neutral Higgs boson, $H$, in the surviving parameter space in section 5 . We then conclude in section 6 .

\section{The Model}

Here we give a brief overview of the 2HDM-II and we set our notation. We take the two scalar doublets $\Phi_{1}$ and $\Phi_{2}$ to have hypercharge $1 / 2$. They can be expanded as

$$
\Phi_{j}=\left(\begin{array}{c}
\phi_{j}^{+} \\
\left(v_{j}+\rho_{j}+i \eta_{j}\right) / \sqrt{2}
\end{array}\right) .
$$

The Yukawa coupling to fermions are given by

$$
\mathcal{L}_{y u k}=-y_{u} \bar{q}_{L}\left(i \sigma^{2} \Phi_{2}^{*}\right) u_{R}-y_{d} \bar{q}_{L} \Phi_{1} d_{R}-y_{e} \bar{e}_{L} \Phi_{1} e_{R}+\text { h.c. }
$$

Expanding out the CP-even neutral scalar sector we obtain

$$
\mathcal{L}_{y u k}=-\frac{M_{u}}{v \sin \beta} \bar{u} u \rho_{2}-\frac{M_{d}}{v \cos \beta} \bar{d} d \rho_{1}-\frac{M_{e}}{v \cos \beta} \bar{e} e \rho_{1},
$$

where $\tan \beta=v_{2} / v_{1}$ and $v^{2}=v_{1}^{2}+v_{2}^{2}$. The two CP-even neutral scalars mix with each other. The mass eigenstates are given by

$$
\left(\begin{array}{l}
\rho_{1} \\
\rho_{2}
\end{array}\right)=\left(\begin{array}{cc}
\cos \alpha & \sin \alpha \\
-\sin \alpha & \cos \alpha
\end{array}\right)\left(\begin{array}{l}
H \\
h
\end{array}\right)
$$

where $h$ is the lighter eigenstate to be identified with the observed $125 \mathrm{GeV}$ Higgs-like particle. In terms of these mass eigenstates, we find

$$
\begin{aligned}
\mathcal{L}_{y u k}= & -\frac{M_{u}}{v}\left(\frac{\cos \alpha}{\sin \beta}\right) \bar{u} u h-\frac{M_{d}}{v}\left(\frac{\sin \alpha}{\cos \beta}\right) \bar{d} d h-\frac{M_{e}}{v}\left(\frac{\sin \alpha}{\cos \beta}\right) \bar{e} e h \\
& +\frac{M_{u}}{v}\left(\frac{\sin \alpha}{\sin \beta}\right) \bar{u} u H-\frac{M_{d}}{v}\left(\frac{\cos \alpha}{\cos \beta}\right) \bar{d} d H-\frac{M_{e}}{v}\left(\frac{\cos \alpha}{\cos \beta}\right) \bar{e} e H
\end{aligned}
$$

The couplings of these two eigenstate $h$ and $H$ to gauge bosons can be obtained from the kinetic terms of $\Phi_{1}$ and $\Phi_{2}$. They are

$$
\begin{aligned}
& \frac{2 M_{W}^{2}}{v} W_{\mu}^{+} W^{-\mu} h \sin (\alpha+\beta)+\frac{M_{Z}^{2}}{v} Z_{\mu} Z^{\mu} h \sin (\alpha+\beta) \\
& \quad+\frac{2 M_{W}^{2}}{v} W_{\mu}^{+} W^{-\mu} H \cos (\alpha+\beta)+\frac{M_{Z}^{2}}{v} Z_{\mu} Z^{\mu} H \cos (\alpha+\beta)
\end{aligned}
$$




\subsection{Scalar Sector}

The scalar sector of the $2 \mathrm{HDM}$ is the most model dependent part. Here we will focus on the simplest scalar potential consistent with CP symmetry

$$
\begin{gathered}
V\left(\Phi_{1}, \Phi_{2}\right)=m_{11}^{2}\left|\Phi_{1}\right|^{2}+m_{22}^{2}\left|\Phi_{2}\right|^{2}+\frac{\lambda_{1}}{2}\left(\left|\Phi_{1}\right|^{2}\right)^{2}+\frac{\lambda_{2}}{2}\left(\left|\Phi_{2}\right|^{2}\right)^{2}+\lambda_{3}\left|\Phi_{1}\right|^{2}\left|\Phi_{2}\right|^{2} \\
+\lambda_{4}\left|\Phi_{1}^{\dagger} \Phi_{2}\right|^{2}+\frac{\lambda_{5}}{2}\left[\left(\Phi_{1}^{\dagger} \Phi_{2}\right)^{2}+\left(\Phi_{2}^{\dagger} \Phi_{1}\right)^{2}\right]
\end{gathered}
$$

It is more convenient to characterize the scalar sector by their physical masses and the mixing angles- $M_{h}, M_{H}, M_{A}, M_{H^{ \pm}}, \alpha, \tan \beta$ :

$$
\begin{aligned}
\sin ^{2} \alpha M_{h}^{2}+\cos ^{2} \alpha M_{H}^{2} & =\frac{v^{2}}{1+\tan ^{2} \beta} \lambda_{1}, \\
\cos ^{2} \alpha M_{h}^{2}+\sin ^{2} \alpha M_{H}^{2} & =\frac{\tan ^{2} \beta}{1+\tan ^{2} \beta} v^{2} \lambda_{2}, \\
\left(M_{h}^{2}-M_{H}^{2}\right) \cos \alpha \sin \alpha & =\left(\lambda_{3}+\lambda_{4}+\lambda_{5}\right) \frac{\tan \beta}{1+\tan ^{2} \beta} v^{2}, \\
M_{A}^{2} & =-\lambda_{5} v^{2}, \\
M_{H^{ \pm}}^{2} & =-\frac{1}{2}\left(\lambda_{4}+\lambda_{5}\right) v^{2}=M_{A}^{2}+\frac{1}{2}\left(\lambda_{5}-\lambda_{4}\right) v^{2} .
\end{aligned}
$$

The set of parameters we use in our parameter-space scan consists of $\alpha, \tan \beta, M_{h}$, $M_{H}, M_{A}$ and $M_{H^{ \pm}}$. We identify the light CP-even scalar, h, with the observed $125 \mathrm{GeV}$ resonance. We do not discuss the alternative hypothesis, that the heavy CP- even scalar is identified with the $125 \mathrm{GeV}$ resonance. The fit to higgs data cannot distinguish between these hypothesis because the couplings of $H$ are the same as those for $h$ after $\alpha \rightarrow \alpha+\pi / 2$. Hence, for the remaining part of this work we set $M_{h}=125 \mathrm{GeV}$.

The scalar potential in equation (2.7) posses a discrete $Z_{2}$ symmetry forbidding terms with odd power of $\Phi_{1}$ or $\Phi_{2}$. This is the defining symmetry of the type II model, designed to avoid flavor changing neutral interactions at tree level [25]. It is a discrete symmetry, rather than continuous Peccei-Quinn U(1)-symmetry [26], to avoid a light axion $[27,28]$. It is conceivable that the symmetry is broken softly by adding to the potential the term $m_{12}^{2} \Phi_{1}^{\dagger} \Phi_{2}+$ h.c.. Such a term would add $m_{12}^{2}\{\tan \beta$, $\cot \beta,-1$, $1 /(\sin \beta \cos \beta), 1 /(\sin \beta \cos \beta)\}$, respectively, to the relations in equation (2.8). We will not pursue this possibility in this paper but we will discuss briefly the effect of this term on the viable parameter space in section 4.3 . 


\section{$3 \quad$ Fit to the Higgs Data}

The mass parameters $M_{H}, M_{A}$ and $M_{H^{ \pm}}$affect Higgs data observables only through suppressed radiative corrections. We determine the VEV ratio $\tan \beta$ and the neutral scalar mixing angle $\alpha$ from all the reported Higgs data.

Experimental data are reported in terms of a signal strength, $\mu$, defined as

$$
\mu \equiv \frac{\sigma}{\sigma^{S M}} \frac{B r}{B r^{S M}} .
$$

When the signal strength is not directly reported by the experimental collaboration, we extract it from the reported $95 \%$ exclusion limit following the procedure given in Ref. [29]. Here we briefly review the procedure and refer the reader to the reference for details. $^{2}$ The experiments report the upperbound on the rate at $95 \%$ C.L., $R_{o b s}$, and the expected upperbound at $95 \%$ C.L. in the absence of the Higgs boson, $R_{e x p}$. The signal strength and its uncertainty, $\sigma$, are given by

$$
\mu=R_{\text {obs }}-R_{\text {exp }}, \quad \sigma=\frac{R_{o b s}}{1.96} .
$$

We collect the signal strengths for each search channel in Table 1.

\subsection{Production Cross-sections and Branching Ratios}

Here we work out the Higgs production cross-section and branching ratios for a nonstadard model Higgs coupling. At tree-level, the non-standard Higgs couplings to SM fields can be characterized by rescaling coefficients, $c_{i}$ 's, relative to the standard model higgs couplings as follows

$$
\mathcal{L}_{h}=c_{V} \frac{h}{v}\left(2 m_{W}^{2} W_{\mu}^{+} W_{\mu}^{-}+m_{Z}^{2} Z_{\mu} Z_{\mu}\right)-c_{t} \frac{h}{v} m_{t} \bar{t} t-c_{b} \frac{h}{v} m_{b} \bar{b} b-c_{c} \frac{h}{v} m_{c} \bar{c} c-c_{\tau} \frac{h}{v} m_{\tau} \bar{\tau} \tau
$$

In this work we assume that other scalars are sufficiently heavy that their effects on the $125 \mathrm{GeV}$ Higgs boson decay channels are negligible.

The main production channels considered here are gluon fusion $(\mathrm{ggF})$, vector boson fusion $(\mathrm{VBF})$, vector boson associated production (Vh) and $t \bar{t} h$ production. These production cross-sections are given in terms of the SM ones by

$$
\frac{\sigma_{g g F}}{\sigma_{g g F}^{S M}}=\left|\frac{1.03 c_{t}-0.05 c_{b}}{1.03-0.05}\right|^{2}, \quad \frac{\sigma_{V B F}}{\sigma_{V B F}^{S M}}=\frac{\sigma_{V h}}{\sigma_{V h}^{S M}}=\left|c_{V}\right|^{2}, \quad \frac{\sigma_{t \bar{t} h}}{\sigma_{t \bar{t} h}^{S M}}=\left|c_{t}\right|^{2}
$$

\footnotetext{
${ }^{2} \mathrm{~A}$ more refined procedure for extracting the signal strength was formulated in Ref. [30]. The two methods give comparable results.
} 


\begin{tabular}{|l|c|c|}
\hline Channel & Signal Strength $(\mu)$ & Reference \\
\hline ATLAS $\gamma \gamma, 7 \mathrm{TeV}$ & $1.6_{-0.8}^{+0.9}$ & {$[31]$} \\
\hline CMS $\gamma \gamma$, dijet-tagged, $7 \mathrm{TeV}$ & $4.21 \pm 2.04$ & {$[32]$} \\
\hline CMS $\gamma \gamma$, untagged $0,7 \mathrm{TeV}$ & $3.15 \pm 1.82$ & {$[32]$} \\
\hline CMS $\gamma \gamma$, untagged $1,7 \mathrm{TeV}$ & $0.66 \pm 0.95$ & {$[32]$} \\
\hline CMS $\gamma \gamma$, untagged $2,7 \mathrm{TeV}$ & $0.73 \pm 1.15$ & {$[32]$} \\
\hline CMS $\gamma \gamma$, untagged $3,7 \mathrm{TeV}$ & $1.53 \pm 1.61$ & {$[32]$} \\
\hline CMS $\gamma \gamma$, dijet-tight, $8 \mathrm{TeV}$ & $1.32 \pm 1.57$ & {$[32]$} \\
\hline CMS $\gamma \gamma$, dijet-loose, $8 \mathrm{TeV}$ & $-0.61 \pm 2.03$ & {$[32]$} \\
\hline CMS $\gamma \gamma$, untagged $0,8 \mathrm{TeV}$ & $1.46 \pm 1.24$ & {$[32]$} \\
\hline CMS $\gamma \gamma$, untagged $1,8 \mathrm{TeV}$ & $1.51 \pm 1.03$ & {$[32]$} \\
\hline CMS $\gamma \gamma$, untagged $2,8 \mathrm{TeV}$ & $0.95 \pm 1.15$ & {$[32]$} \\
\hline CMS $\gamma \gamma$, untagged $3,8 \mathrm{TeV}$ & $3.78 \pm 1.77$ & {$[32]$} \\
\hline ATLAS $\gamma \gamma, 8 \mathrm{TeV}$ & $1.6 \pm 0.32$ & {$[13]$} \\
\hline ATLAS ZZ, 7 TeV & $1.4_{-0.8}^{+1.3}$ & {$[33]$} \\
\hline CMS ZZ, 7 TeV & $0.6_{-0.6}^{+0.9}$ & {$[34]$} \\
\hline ATLAS ZZ, combine $7 \& 8 \mathrm{TeV}$ & $1.5 \pm 0.6$ & {$[13]$} \\
\hline CMS ZZ, combine $7 \& 8 \mathrm{TeV}$ & $0.91_{-0.24}^{+0.30}$ & {$[14]$} \\
\hline ATLAS WW, $7 \mathrm{TeV}$ & $0.1_{-0.6}^{+0.7}$ & {$[35]$} \\
\hline CMS WW, 7 TeV & $0.4 \pm 0.6$ & {$[34]$} \\
\hline ATLAS WW, $8 \mathrm{TeV}$ & $1.45 \pm 0.56$ & {$[10]$} \\
\hline CMS WW, combine $7 \& 8 \mathrm{TeV}$ & $0.76 \pm 0.21$ & {$[15]$} \\
\hline CMS $b \bar{b}, 7 \mathrm{TeV}$ & $1.2_{-1.7}^{+2.1}$ & {$[34]$} \\
\hline CMS $b \bar{b}, 8 \mathrm{TeV}$ & $1.07 \pm 0.62$ & {$[11]$} \\
\hline Tevatron $b \bar{b}$ & $2.0 \pm 0.7$ & {$[12]$} \\
\hline ATLAS $\tau \bar{\tau}, 8 \mathrm{TeV}$ & $0.7 \pm 0.7$ & {$[36]$} \\
\hline CMS $\tau \bar{\tau}, 8 \mathrm{TeV}$ & $0.88 \pm 0.50$ & {$[11]$} \\
\hline
\end{tabular}

Table 1: The signal strengths and the corresponding error for the Higgs data used in the fit.

The rescaling factors for the partial decays widths are

$$
\begin{gathered}
\Gamma_{b b}=\left|c_{b}\right|^{2} \Gamma_{b b}^{S M}, \quad \Gamma_{c c}=\left|c_{c}\right|^{2} \Gamma_{c c}^{S M}, \quad \Gamma_{\tau \tau}=\left|c_{\tau}\right|^{2} \Gamma_{\tau \tau}^{S M}, \quad \Gamma_{V V}=\left|c_{V}\right|^{2} \Gamma_{V V}^{S M}, \\
\frac{\Gamma_{g g}}{\Gamma_{g g}^{S M}}=\left|\frac{1.03 c_{t}-0.05 c_{b}}{1.03-0.05}\right|^{2}, \quad \frac{\Gamma_{\gamma \gamma}}{\Gamma_{\gamma \gamma}^{S M}}=\left|\frac{\frac{2}{9} 1.03 c_{t}-1.04 c_{V}}{\frac{2}{9} 1.03-1.04}\right|^{2},
\end{gathered}
$$


where we have assumed that the loop-induced, charged Higgs contribution to $\Gamma_{\gamma \gamma}$ is negligible. This assumption can be justified once we include experimental constraints from section 4.2. The rescaling factors for the $2 \mathrm{HDM}-\mathrm{II}$ are given by

$$
c_{t}=c_{c}=\frac{\cos \alpha}{\sin \beta}, \quad c_{b}=c_{\tau}=\frac{\sin \alpha}{\cos \beta}, \quad c_{V}=\sin (\alpha+\beta) .
$$

For completeness we include the rescaling of the partial decay width for $h \rightarrow \gamma Z$

$$
\frac{\Gamma_{\gamma Z}}{\Gamma_{\gamma Z}^{S M}}=\left|\frac{-(0.38+0.37 i) 0.86 c_{t}-(3.32+3.98 i) c_{V}}{-(0.38+0.37 i) 0.86-(3.32+3.98 i)}\right|^{2} .
$$

The general expressions for the loop-induced decay of a neutral scalar boson are collected in appendix A.

\subsection{Global Fit}

We determine the best fit values of $\tan \beta$ and $\alpha$ using all the reported Higgs data from the Tevatron, LHC $7 \mathrm{TeV}$ and LHC $8 \mathrm{TeV}$ runs. The theoretical SM Higgs boson predictions for the cross-sections and branching ratios are taken from the LHC Higgs Cross-section Working Group [37]. We obtain best fit values $\tan \beta \simeq 0.01$ and $\alpha \simeq 1.56$. At this small value of $\tan \beta$ the top Yukawa coupling is non-perturbative at the weak scale. Thus the best fit value doesn't seem to correspond to viable model parameters.

Assuming a Gaussian distribution around the best fit values, we determine the region of parameter-space consistent within 1 - and $2-\sigma$ with the best fit values. The result is shown in figure 1 . The dashed line indicates the decoupling limit, $\alpha+\beta=$ $\pi / 2$, where the couplings are SM-like (up to a possible sign flip for the down Yukawa couplings). The figure is truncated at $\alpha<0.5$, since for larger values $\tan \beta$ decreases, forcing the top Yukawa coupling into a non-perturbative regime.

\section{Bounds on Parameter Space}

\subsection{Theoretical Constraints}

We impose constraints amounting to the potential being bounded from below. To this end the couplings must satisfy [24]

$$
\begin{aligned}
\lambda_{1}, \lambda_{2} & >0, \\
\lambda_{3} & >-\sqrt{\lambda_{1} \lambda_{2}}, \\
\lambda_{3}+\lambda_{4}-\left|\lambda_{5}\right| & >-\sqrt{\lambda_{1} \lambda_{2}},
\end{aligned}
$$

at all scales up to the cutoff scale $\Lambda$. 


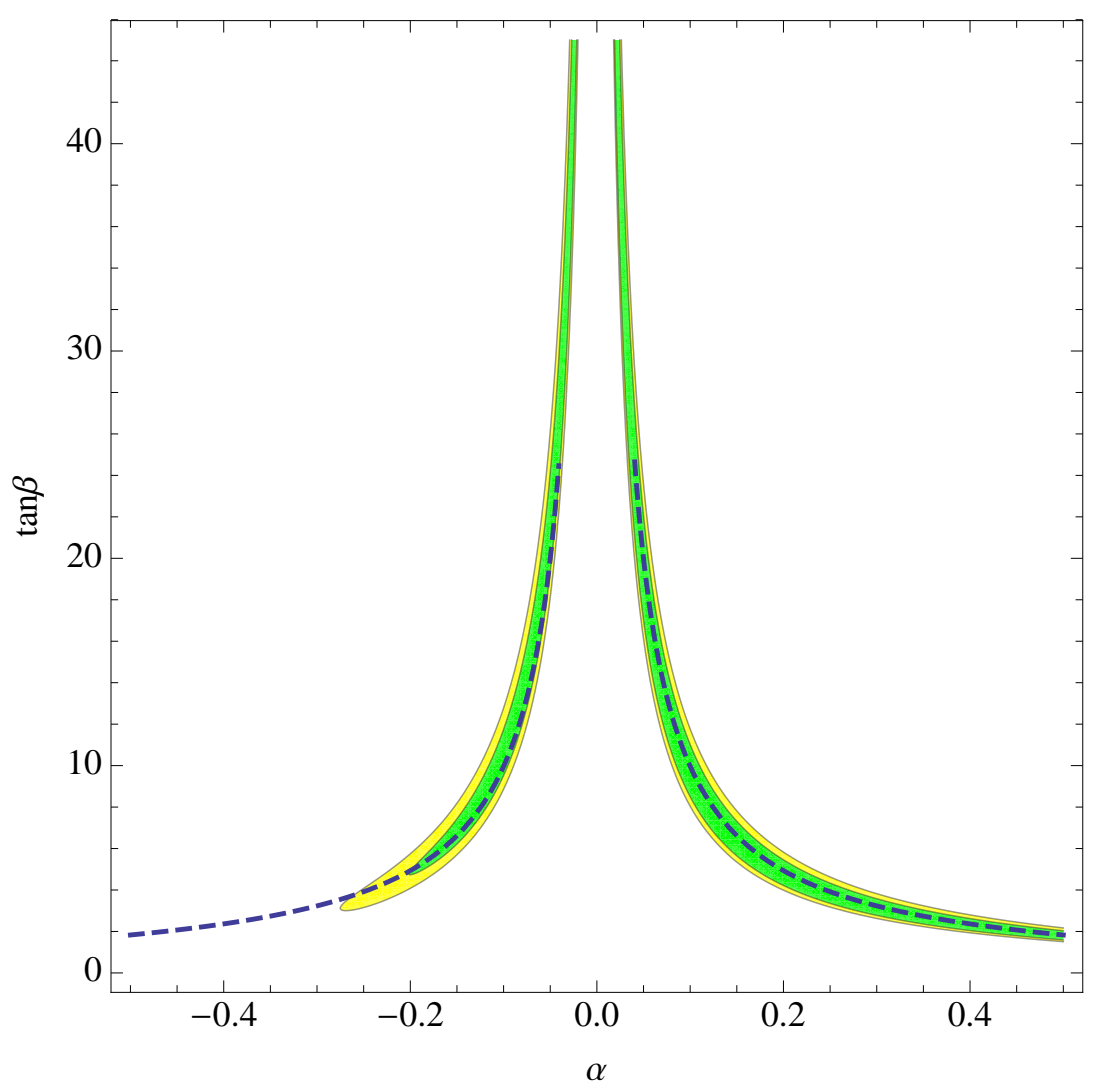

Figure 1: The region of parameter space within 1- and 2- $\sigma$ of the best fit values. The dashed line is the decoupling limit, $\alpha+\beta= \pm \pi / 2$, where the couplings are SM-like (up to a possible sign flip for the down Yukawa couplings).

We also impose the following perturbativity constraint on the couplings

$$
\frac{y_{i}^{2}}{4 \pi} \leq 1, \quad \frac{\lambda_{i}}{4 \pi} \leq 1 .
$$

We insist on these constraints up to the cutoff scale for all the Yukawa and scalar couplings. We list the beta-functions used in evolving the coupling constants in appendix B.

\subsection{Experimental Bounds}

A wealth of experimental data, particularly from precision measurements, places strong constraints on the spectrum of the 2HDM-II. A newly published result on a direct search for the charged Higgs at LEP yields the $95 \%$ CL lower bound $M_{H^{ \pm}} \geq 80 \mathrm{GeV}$ [38]. At present there is no lower bound on the charged Higgs mass from the Tevatron or LHC 
data. A much tighter constraint on the charged Higgs mass can be deduced from rare decay processes. By analyzing the branching ratio $\operatorname{Br}\left(\bar{B} \rightarrow X_{s} \gamma\right)$, Ref. [39] obtained the bound $M_{H^{ \pm}} \geq 380 \mathrm{GeV}$ at $95 \%$ confidence level. A direct search at LEP places a $95 \%$ limit $M_{A} \gtrsim 93 \mathrm{GeV}$ for the MSSM CP-odd Higgs, $A$ [40]. However this limit doesn't apply to the 2HDM case studied here. Nevertheless, we employed this bound in in the rest of the paper. The reader should keep in mind that $M_{A} \lesssim 93 \mathrm{GeV}$ is not experimentally excluded.

Electroweak precision measurements also place strong constraints on the spectrum of a 2HDM. We concentrate on the oblique $S$ and $T$ parameters. In the Standard Model, the best fit values for the $S$ and $T$ parameters for $M_{h} \sim 125 \mathrm{GeV}$ and $M_{t}=173$ $\mathrm{GeV}$, as well as their correlation matrix $\left(M_{\text {corr }}\right)$ are [41].

$$
\begin{aligned}
& S=0.03 \pm 0.10, \\
& T=0.05 \pm 0.12,
\end{aligned} \quad M_{\text {corr }}=\left(\begin{array}{cc}
1 & 0.891 \\
0.891 & 1
\end{array}\right) .
$$

Additional contribution to the $S$ and $T$ parameters from the heavy Higgs bosons are given in Ref. [16].

\subsection{Viable Parameter Space}

In this section we present the result of our parameter space scan consistent with both the Higgs data and the experimental and theoretical constraints discussed above. A point in parameter space is a set of values of the parameters $M_{H}, M_{A}, M_{H^{ \pm}}, \alpha, \tan \beta$, as discussed in section 2.1. For every parameter point consistent with the experimental constraints of 4.2, we determine the corresponding scalar and Yukawa couplings using equations (2.5) and (2.8). These couplings are then evolved numerically from the weak scale, $v_{w}$, to the cut-off scale, $\Lambda$, using beta-functions listed in appendix B. Finally the couplings are checked against the theoretical constraints of section 4.1.

The interplay between the experimental and theoretical bounds on the spectrum can be easily understood. Intuitively, experimental bounds- $\operatorname{Br}\left(\bar{B} \rightarrow X_{s} \gamma\right)$ and electroweak precision data tend to drive the mass of the Higgs bosons heavy in order to leave a small imprint on low energy observables. At the same time, the more massive the spectrum is, the larger the scalar couplings. Perturbativity constraints limit how large these scalar couplings can be, hence limit from above the Higgs spectrum of the theory $^{3}$. Combining these experimental and theoretical considerations, we find that with the cut-off scale $(\Lambda)$ at $2 \mathrm{TeV}$, there is no viable parameter space. Reducing $\Lambda$ to $1 \mathrm{TeV}$ opens up a small parameter space for small values of $\tan \beta$. Thus, for the rest of this work we will take $\Lambda=1 \mathrm{TeV}$. The viable spectrum is shown in figure 2 .

\footnotetext{
${ }^{3}$ This conclusion is relaxed somewhat if we allow the $Z_{2}$ symmetries to be broken softly, see section 2.1 .
} 

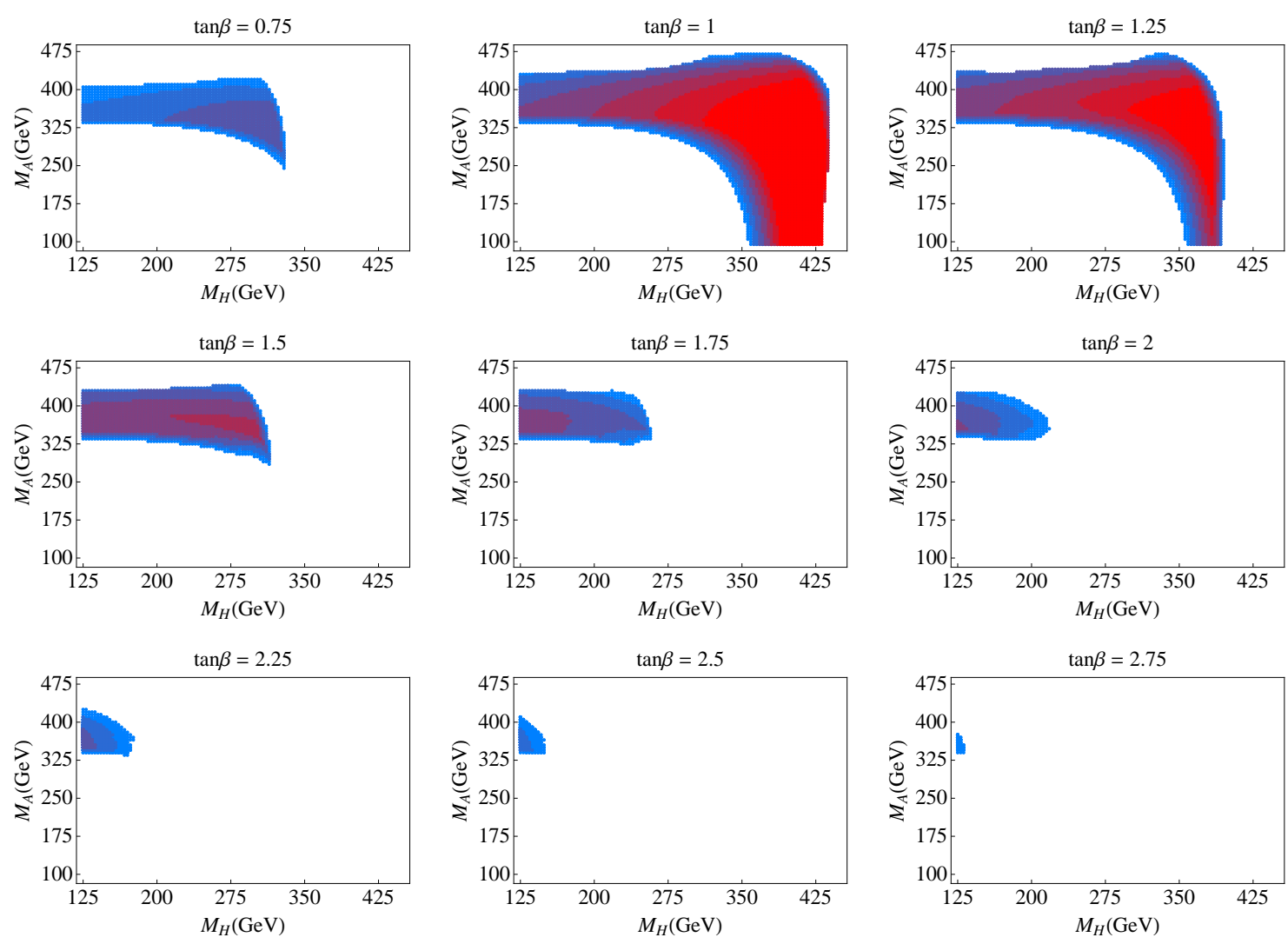

Figure 2: The viable particle spectrum for $0.75 \leq \tan \beta \leq 2.75$. The spectrum depends non-trivially on the charged Higgs mass $\left(M_{H^{ \pm}}\right)$. In each plot the viable parameter space in $M_{H^{-}} M_{A}$ plane shrinks as $M_{H^{ \pm}}$increases. $M_{H^{ \pm}}=380$ (420) $\mathrm{GeV}$ corresponds to the color blue (red).

We end this section with a brief discussion of the sensitivity of the viable parameter space on the choice of perturbativity condition, eg. equation (4.2). Had we imposed instead that all the reduced couplings remain less than $1 / 2$, there would be no viable parameter space. We find that relaxing the perturbativity constraint from $1 / 2$ to $3 / 4$ opens up a small viable parameter space for the cases $\tan \beta=1,1.25$. Relaxing this constraint further to 1 leads us to the viable parameter space that we have in figure 2 .

\section{Phenomenology Of The Other Higgs Bosons}

In this section we study the phenomenology of the neutral CP-even and CP-odd Higgs bosons, $H$ and $A$. We will focus on their production cross-sections and branching ratios. For a wide range of production and decay channels, we can deduce the cross-sections 
$8 \mathrm{TeV}, \tan \beta=1.0$
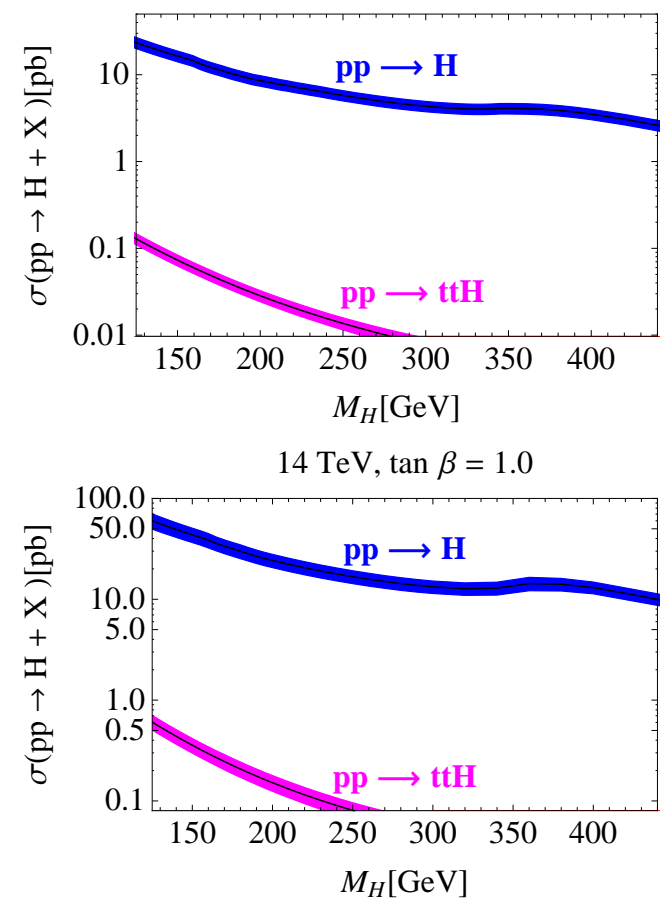

$8 \mathrm{TeV}, \tan \beta=1.5$
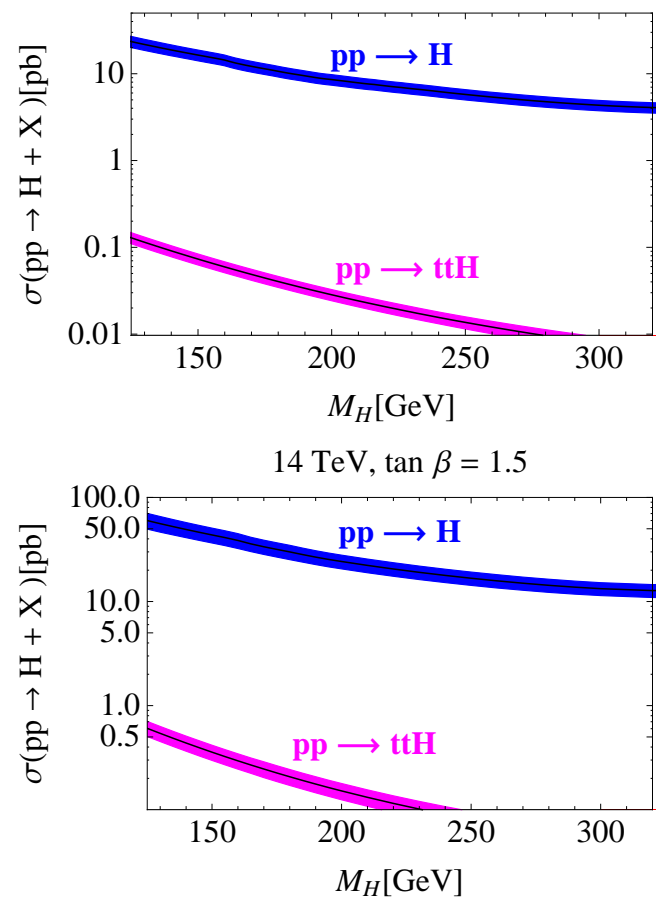

Figure 3: The heavy CP-even Higgs boson production cross-sections. For each plot, $\alpha$ is taken to be $0.78(0.58)$ for $\tan \beta=1.0$ (1.5). This choice of $\alpha$ minimizes $\chi^{2} \mid \tan \beta$ for a given value of $\tan \beta$.

and branching ratios by scaling from the corresponding quantities for the SM Higgs boson. When this scaling procedure is not available, we compute the corresponding quantity at leading order.

\subsection{Phenomenology of the CP-even $H$}

The couplings of the heavy CP-even Higgs boson $H$ to the SM fermions and gauge bosons are modified with respect to the SM Higgs boson couplings by the rescaling factors

$$
c_{t}^{H}=c_{c}^{H}=\frac{-\sin \alpha}{\sin \beta}, \quad c_{b}^{H}=c_{\tau}^{H}=\frac{\cos \alpha}{\cos \beta}, \quad c_{V}^{H}=\cos (\alpha+\beta) .
$$

The $H$ production cross-sections can be readily obtained by rescaling the corresponding calculations for SM Higgs boson production. The CP-even $H$ boson production crosssections for the 8 and $14 \mathrm{TeV}$ LHC are shown in figure 3. Note that since the mixing angles $\alpha$ and $\beta$ are close to the decoupling limit, the $H$ production cross-sections in the vector boson fusion and the $W$ - and $Z$-associated production channels are suppressed. 

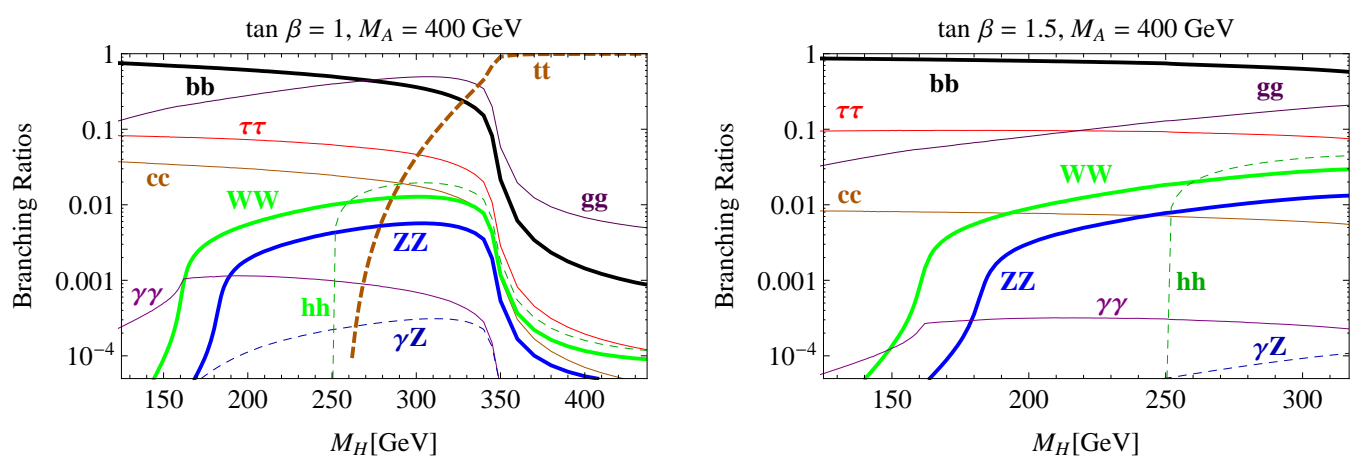

Figure 4: The heavy CP-even Higgs boson branching ratios for the case of heavy $M_{A}$. For each plot, $\alpha$ is taken to be 0.78 (0.58) for $\tan \beta=1.0$ (1.5). This choice of $\alpha$ minimizes $\left.\chi^{2}\right|_{\tan \beta}$ for a given value of $\tan \beta$.

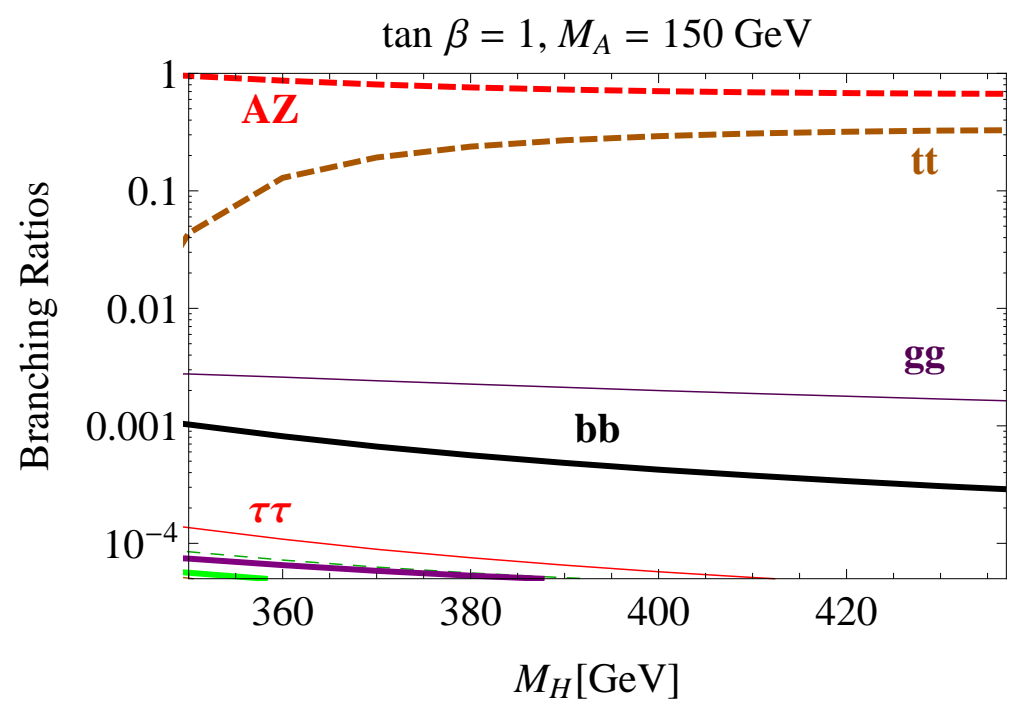

Figure 5: The heavy CP-even Higgs boson branching ratios for the case of light $M_{A}$. Here $\alpha$ is taken to be 0.78 . This choice of $\alpha$ minimizes $\left.\chi^{2}\right|_{\tan \beta}$ for a given value of $\tan \beta$.

In a large portion of the available parameter space $M_{H}$ is lighter than $2 M_{h}, M_{A}$ and $M_{H^{ \pm}}$; see figure 2. When this is the case all the available decay channels for the heavy Higgs boson, $H$, are the same as for the SM Higgs boson. The branching ratios can be obtained by a simple rescaling from the corresponding values of the SM Higgs boson. When $M_{H}$ is sufficiently heavy, the decays $H \rightarrow h h, H \rightarrow A Z$ and $H \rightarrow A A$ 
become available. The partial widths $\operatorname{are}^{4}$

$$
\begin{aligned}
\Gamma(H \rightarrow \phi \phi) & =\frac{G_{F}}{8 \sqrt{2} \pi} \frac{\bar{\lambda}_{H \phi \phi}^{2}}{M_{H}} \sqrt{1-\frac{4 M_{\phi}^{2}}{M_{H}^{2}}} \\
\Gamma(H \rightarrow A Z) & =\frac{G_{F}}{8 \sqrt{2} \pi} \sin ^{2}(\alpha+\beta)\left[\frac{\left(M_{H}^{2}-\left(M_{Z}-M_{A}\right)^{2}\right)\left(M_{H}^{2}-\left(M_{Z}+M_{A}\right)^{2}\right)}{M_{H}^{2}}\right]^{3 / 2},
\end{aligned}
$$

where $G_{F}$ is the Fermi constant, $\phi=h, A$ and

$$
\begin{aligned}
\bar{\lambda}_{H h h} & =\frac{\left(2 M_{h}^{2}+M_{H}^{2}\right) \cos (\alpha+\beta) \sin (2 \alpha)}{2 \sin \beta \cos \beta} \\
\bar{\lambda}_{H A A} & =\frac{\left(3 M_{H}^{2}+2 M_{A}^{2}\right) \sin (\alpha-\beta)+\left(M_{H}^{2}-2 M_{A}^{2}\right) \sin (\alpha+3 \beta)}{4 \sin \beta \cos \beta} .
\end{aligned}
$$

The branching fractions for $H$ decays are shown in figure 4 and 5 . Since the mixing angles $\alpha$ and $\beta$ are close to the decoupling limit, the decays into a pair of massive vector bosons are suppressed. When $H \rightarrow A+X$ is kinematically forbidden, the $H$ boson decays predominantly into quarks and gluons; see figure 4 . In this case, it is most likely to observe the $H$-boson in di-photon decays. However, when $H \rightarrow A+X$ is kinematically allowed, the decay mode $H \rightarrow A Z$ becomes the most dominant; see figure 5. As we will show in the next section, the $A$ has sizable branching ratios into $\gamma Z$ and $\gamma \gamma$. Thus it might be possible to observe $H \rightarrow A Z$ in a photon plus four leptons or two photons and two leptons channels.

\subsection{Phenomenology of the CP-odd Boson}

The pseudoscalar $A$ does not couple at tree level to a pair of electroweak gauge bosons. Thus its main production mechanism is via gluon fusion. Its production cross-section can be obtained from that of the Higgs in the Standard Model by rescaling by a factor

$$
r_{g} \simeq\left|\cot \beta \frac{\tau f(\tau)}{\tau+(\tau-1) f(\tau)}\right|^{2},
$$

where $\tau=M_{A}^{2} / 4 M_{t}^{2}$ and the function $f(\tau)$ is defined in appendix A. The production cross-sections of the pseudoscalar $A$ at the LHC with $8 \mathrm{TeV}$ and $14 \mathrm{TeV}$ center-of-mass energy are shown in figure 6.

The CP-odd boson $A$ has a mass $M_{A} \lesssim 470 \mathrm{GeV}$, see figure 2. A light $A$ can decay into a pair of light quark-antiquark as well as into two photons, much like the

\footnotetext{
${ }^{4}$ Our result does not agree with that of Ref [42] whose partial width for $\Phi \rightarrow \phi Z$ does not have a correct mass dimension.
} 

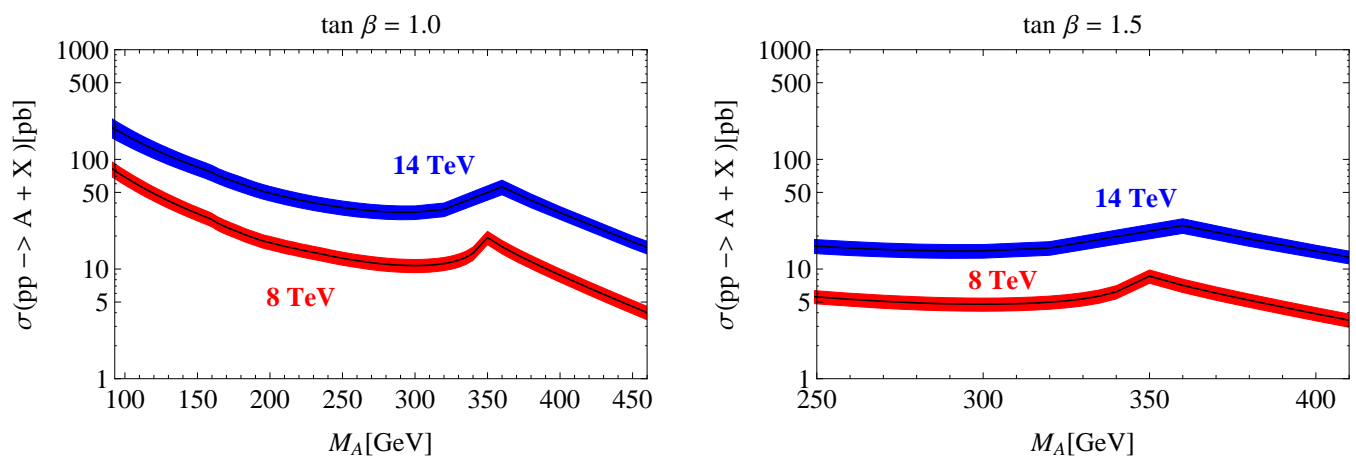

Figure 6: The heavy CP-odd Higgs boson production cross-sections. For each plot, $\alpha$ is taken to be $0.78(0.58)$ for $\tan \beta=1.0$ (1.5). This choice of $\alpha$ minimizes $\left.\chi^{2}\right|_{\tan \beta}$ for a given value of $\tan \beta$.

SM Higgs. For sufficiently large $M_{A}, A$ can also decay into pair consisting of a neutral CP-even scalar boson and a vector boson, $\phi Z$, where $\phi=h, H$. The partial decay widths for $A \rightarrow q \bar{q}$ is

$$
\Gamma(A \rightarrow q \bar{q})=3 \frac{G_{F} M_{q}^{2}}{4 \sqrt{2} \pi} \delta_{q}^{2} M_{A} \sqrt{1-4 M_{q}^{2} / M_{A}^{2}},
$$

where $\delta_{q}=\cot \beta(\tan \beta)$ for up-type (down-type) quark. The partial decay width into $\phi Z$ is given by

$$
\Gamma(A \rightarrow \phi Z)=\frac{G_{F}}{8 \sqrt{2} \pi} \delta_{\phi}^{2}\left[\frac{\left(M_{A}^{2}-\left(M_{Z}-M_{\phi}\right)^{2}\right)\left(M_{A}^{2}-\left(M_{Z}+M_{\phi}\right)^{2}\right)}{M_{A}^{2}}\right]^{3 / 2},
$$

where $\delta_{h}\left(\delta_{H}\right)=\cos (\alpha+\beta)(\sin (\alpha+\beta))$. The loop-induced branching ratios into $g g, \gamma \gamma$ and $\gamma Z$ are given in appendix A. The CP-odd $A$ branching ratios are shown in figure 7 . Note that the suppression of $\operatorname{Br}(A \rightarrow h Z)$ arises because the two mixing angles $\alpha$ and $\beta$ are close to the decoupling limit, see equation (5.6). The dominant decay channels for $A$ are $b \bar{b}$ and $g g$ for a low mass $A$ and $H Z$ and $t \bar{t}$ for a heavy $A$.

It is also interesting to note that for the low mass range below the $t \bar{t}$ and $H Z$ thresholds, the $A$ production cross-section and branching ratios into $\gamma \gamma$ and $\gamma Z$ are enhanced compared to the corresponding Standard Model Higgs boson counterparts. Our estimate of the signal strength in these two modes is shown in figure 8. With the large signal strength for $M_{A} \geq 160 \mathrm{GeV}$, this scenario could be excluded using current Higgs data. However, at the moment both the ATLAS and CMS collaborations only provide the $95 \%$ exclusion limit on the signal strength in these channels up to a mass of $150 \mathrm{GeV}$ [43-46]. 

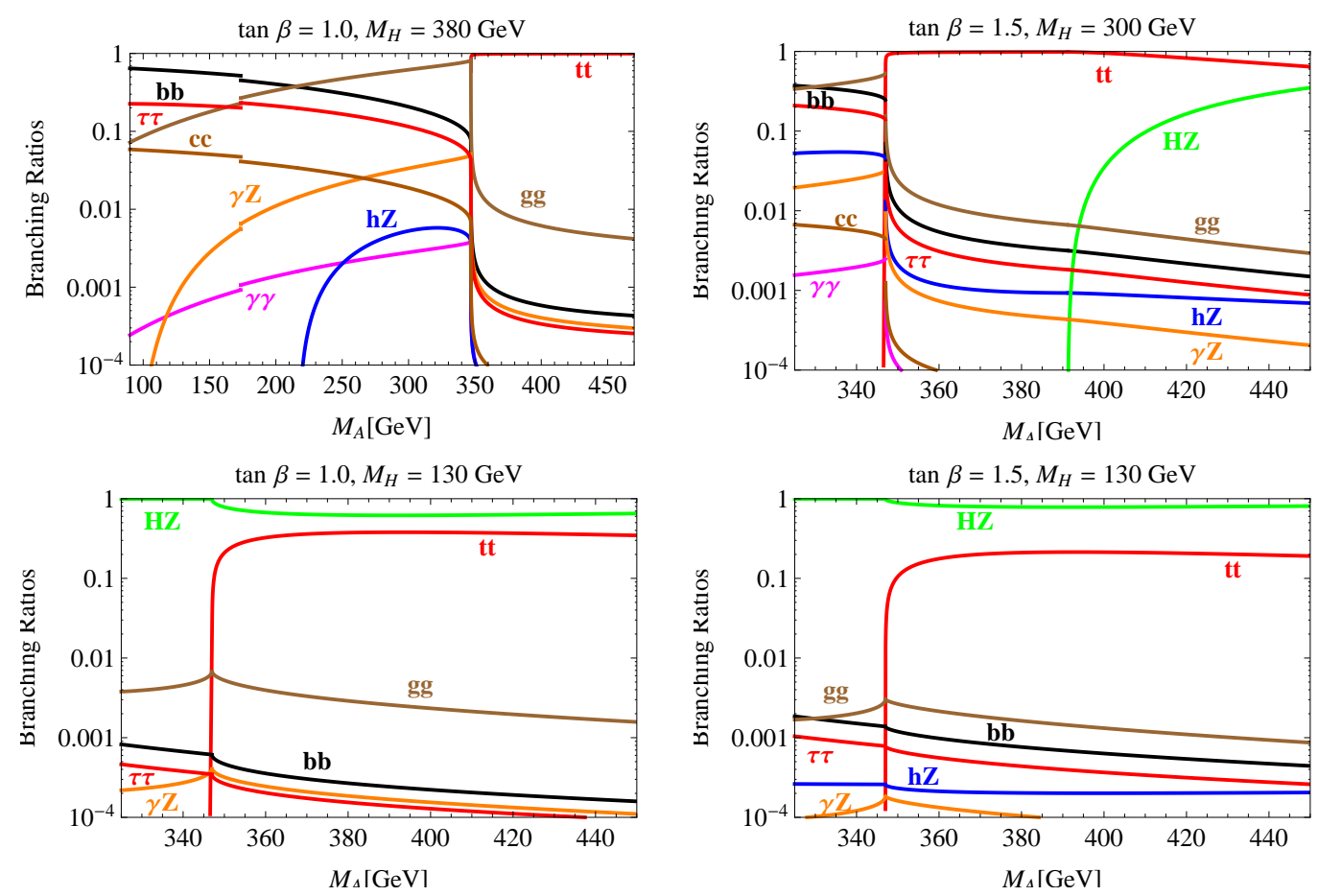

Figure 7: The CP-odd Higgs boson branching ratios. For each plot, $\alpha$ is taken to be 0.78 (0.58) for $\tan \beta=1.0$ (1.5). This choice of $\alpha$ minimizes $\left.\chi^{2}\right|_{\tan \beta}$ for a given value of $\tan \beta$. Diagrams on the top correspond to the case when $M_{H}$ is sufficiently heavy and cannot be in the decay product of $A$. Diagrams on the bottom are when $M_{H}$ is light enough to be in the decay product. For definiteness, we take $M_{H}=130 \mathrm{GeV}$. Here we ignore the effect of near threshold production

\subsection{Phenomenology of the Charged Boson $H^{ \pm}$}

The viable mass of the charged Higgs boson is larger than that of the top-quark. Thus its main production cross-section is from $g \bar{b} \rightarrow H^{+} \bar{t}$ and $g g \rightarrow H^{+} \bar{t} b$ [47]. We use Madgraph to compute the lowest order production cross-sections for both processes. We follow Ref. [48] in keeping the renormalization scale and the PDF factorization scale fixed at $\mu=M_{t}+M_{H^{ \pm}}$. For comparison, we also compute the production cross-section from $q \bar{q} \rightarrow H^{+} \bar{t} b$ where $q$ is the valence quarks. The production cross-sections are shown in figure 9 .

The physical charged Higgs couplings to quarks are given by

$$
\left(\sqrt{2} G_{F}\right)^{1 / 2}\left[\cot \beta\left(m_{u}\right)_{i} \bar{u}_{i} V_{i j}^{*} P_{L} d_{j}+\tan \beta\left(m_{d}\right)_{j} \bar{u}_{i} V_{i j}^{*} P_{R} d_{j}\right] H^{+}+\text {h.c. }
$$

where $m_{u}\left(m_{d}\right)$ is the mass matrix of the up (down) type quarks and $V$ is the CKM 


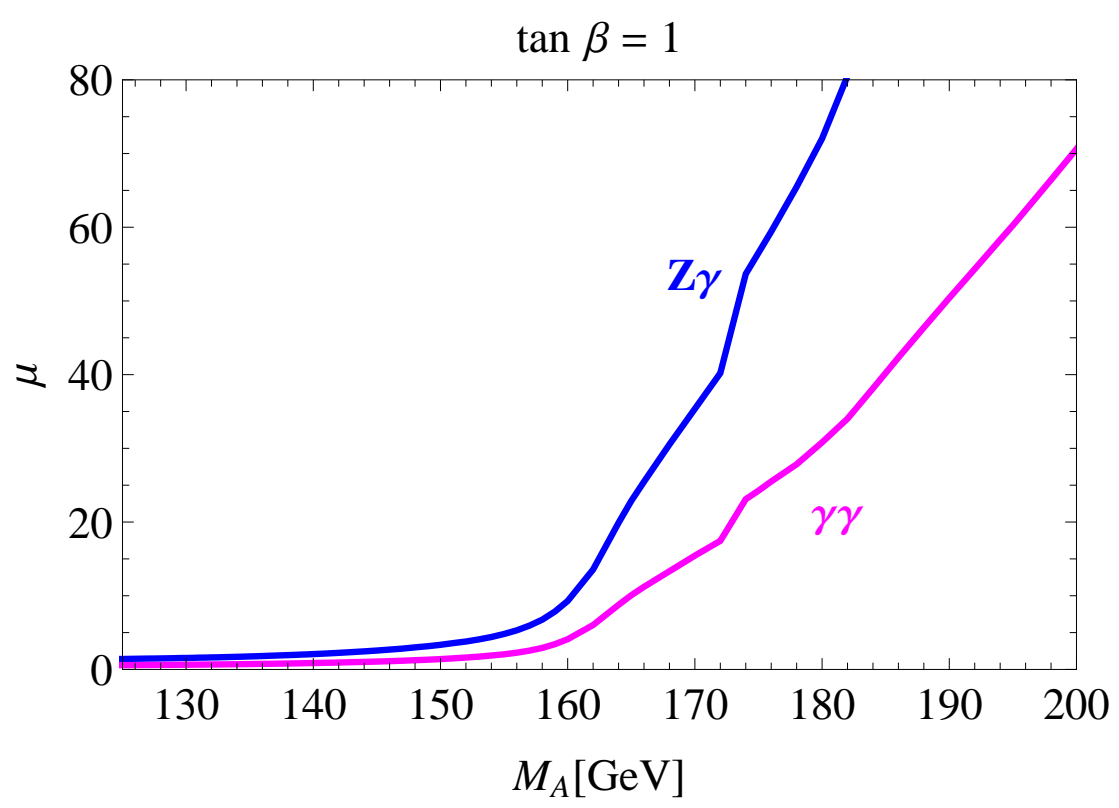

Figure 8: The signal strength $\mu(A \rightarrow \gamma \gamma)$ and $\mu(A \rightarrow \gamma Z)$ for the case $\tan \beta=1$ in the low mass range.

matrix. Thus its partial width $\Gamma\left(H^{+} \rightarrow t \bar{b}\right)$ at leading order is

$$
\begin{aligned}
\Gamma\left(H^{+} \rightarrow t \bar{b}\right)= & \frac{3 G_{F}}{8 \sqrt{2} \pi}\left|V_{t b}\right|^{2} \sqrt{\frac{\left(M_{H^{ \pm}}^{2}-\left(M_{t}^{2}+M_{b}^{2}\right)\right)\left(M_{H^{ \pm}}^{2}-\left(M_{t}^{2}-M_{b}^{2}\right)\right)}{M_{H^{ \pm}}^{2}}} \\
& {\left[\left(1-\frac{M_{t}^{2}}{M_{H^{ \pm}}^{2}}-\frac{M_{b}^{2}}{M_{H^{ \pm}}^{2}}\right)\left[M_{t}^{2} \cot ^{2} \beta+M_{b}^{2} \tan ^{2} \beta\right]+\frac{4 M_{t}^{2} M_{b}^{2}}{M_{H^{ \pm}}^{2}}\right] . }
\end{aligned}
$$

The charged Higgs can also decay into a neutral scalar and a vector. In this case the partial width can be read off from equation (5.6) with the replacement $M_{A} \rightarrow M_{H^{ \pm}}$ and $\delta_{A}=1$. The branching ratios of the charged Higgs boson are shown in figure 10 . Much like for $A \rightarrow h Z$, the branching ratio for $H^{+} \rightarrow h W^{+}$is suppressed because $\alpha$ and $\beta$ are close to the decoupling limit. For a light $H(A)$, the decay $H^{ \pm} \rightarrow H(A) W^{ \pm}$ is dominant. This decay leads to an event with 3 or $4 b$-quarks (depending on the production channel) plus a charged lepton and missing energy which could be searched for at the LHC.

\section{Conclusions and Discussions}

We have performed a parameter scan for the CP conserving 2HDM-II consistent with all the available Higgs data. We take into account theoretical bounds — vacuum stability 

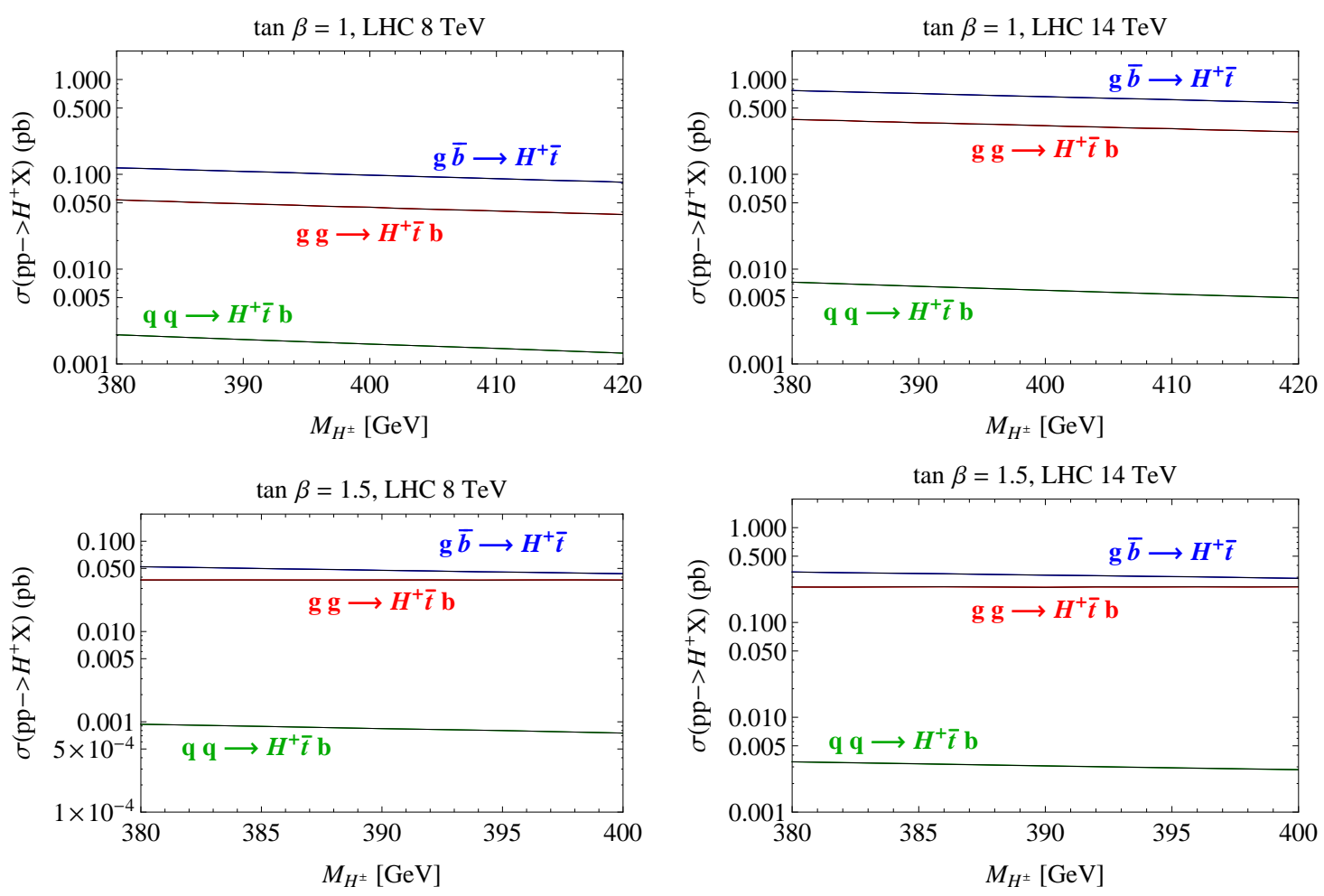

Figure 9: The charged Higgs boson production cross-sections for the case $\tan \beta=$ 1.0 (1.5) for the left (right) plot with $\alpha=0.78(0.58) . \alpha$ is chosen such that it minimizes $\left.\chi^{2}\right|_{\tan \beta}$ for a given value of $\tan \beta$. Diagrams on the top correspond to the 8 $\mathrm{TeV}$ LHC while the bottom are for $14 \mathrm{TeV}$.

and perturbativity of the couplings, as well as experimental bounds from electroweak precision measurements and $\operatorname{Br}\left(\bar{B} \rightarrow X_{s} \gamma\right)$ in our scan. We use a working assumption that the $2 \mathrm{HDM}$-II is a valid low energy effective theory up to a cut-off scale $\Lambda$. We found that for $\Lambda \geq 2 \mathrm{TeV}$, there is no viable parameter space consistent with all the mentioned constraints. However, if we assume $\Lambda=1 \mathrm{TeV}$, only a small parameter space of the 2HDM-II is consistent with all the mentioned constraints. In particular, the ratio of the vacuum expectation value of the two scalar doublets, $\tan \beta$, lies in the range $0.75 \lesssim \tan \beta \lesssim 2.75$.

Our results show that the charged Higgs boson is the most constrained sector of the 2HDM-II. The perturbativity constraint demands $M_{H^{ \pm}} \leq 420 \mathrm{GeV}$ while constraint from $\operatorname{Br}\left(\bar{B} \rightarrow X_{s} \gamma\right)$ pushes $M_{H^{ \pm}} \geq 380 \mathrm{GeV}$. Since the mass of the charged Higgs boson is much heavier than that of the top-quark, its main production mechanism is production in association with the top or in association in the top and the bottom- 

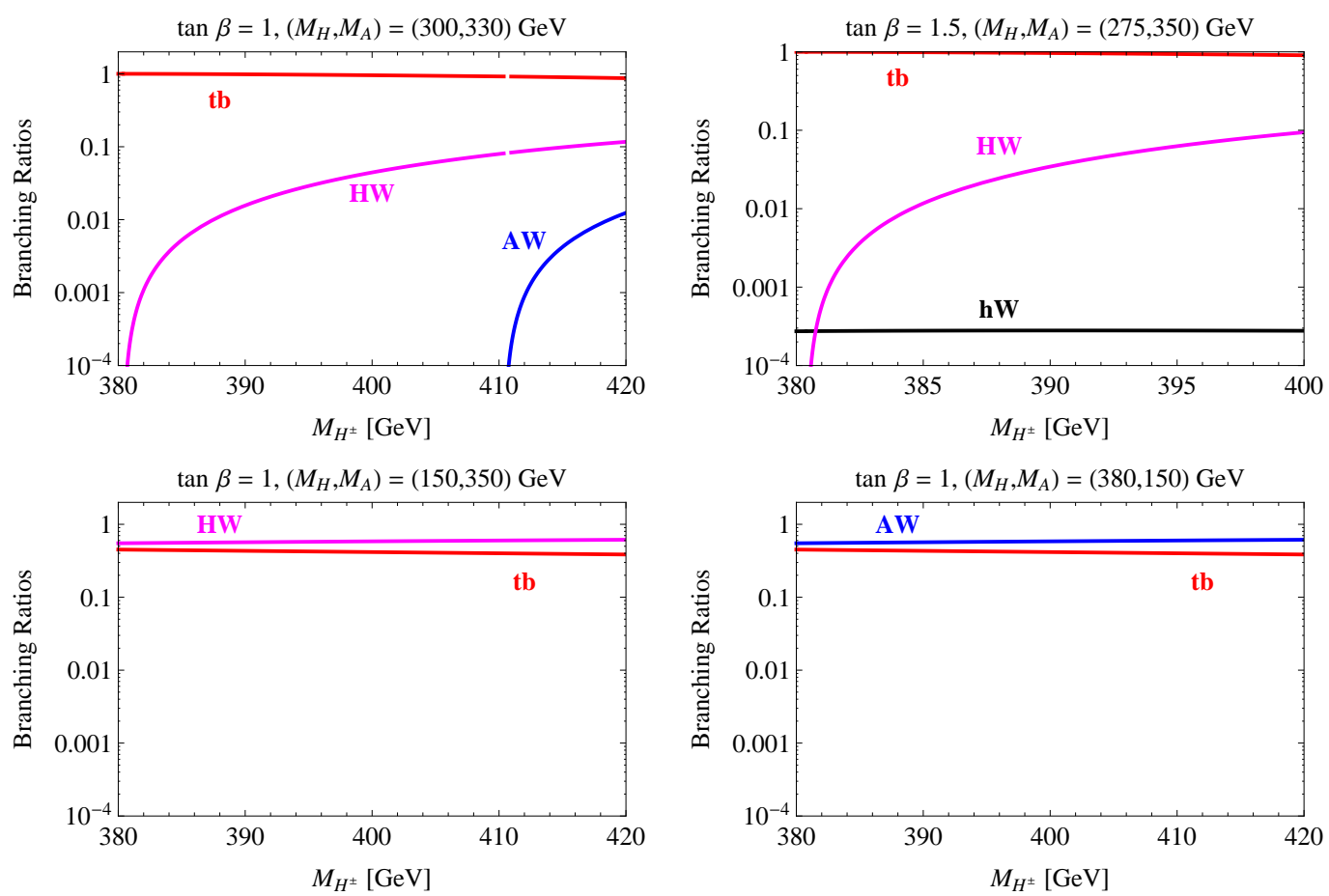

Figure 10: The charged Higgs boson branching ratios for the case $\tan \beta=1.0(1.5)$ for the left (right) plot with $\alpha=0.78(0.58)$. $\alpha$ is chosen such that it minimizes $\chi^{2} \mid \tan \beta$ for a given value of $\tan \beta$. The masses of the neutral Higgs boson are chosen so that they are consistent with the viable parameter space shown in figure 2

quarks. The charged Higgs, once produced, decays mostly into a top- and bottomquark pair which makes its detection difficult. However, in a corner of parameter space where the heavy CP-even $H$ (or the CP-odd $A$ ) is light enough, the decay into $H(A) W$ becomes comparable to the $t b$ channel. The light $H(A)$ then mostly decays into a $b \bar{b}$ pair. This decay chain leads to an event with $3 b$-quarks (or 4 , depending on the production mechanism) plus a charged lepton and missing energy which makes it possible to be searched for at the LHC.

The neutral scalars sector is not as tightly constrained. The masses of both the heavy CP-even Higgs boson, $H$, and the CP-odd, $A$, can take on values in a large range; see figure 2. The production cross-sections for these two particle are sizable at both the $8 \mathrm{TeV}$ and the $14 \mathrm{TeV}$ LHC. Since the mixing angles $\alpha$ and $\beta$ are close to the decoupling limit, the $H$ is mainly produced via gluon fusion. Hence a low mass $H$, where $H$ decays predominantly into $b \bar{b}$, will be difficult to observe at the LHC. However, for a heavy $H$ the decay $H \rightarrow A Z$ becomes available and could lead to spectacular decay signatures 
of one photon and 4 leptons or two photon and leptons if the $A$ decays into $\gamma Z$ or $\gamma \gamma$ and the $Z$ 's decay leptonically.

A low mass pseudo scalar $A$ has an enhanced production cross-section compared to the SM Higgs boson counterpart. Moreover, its branching ratios into $\gamma Z$ and $\gamma \gamma$ are also enhanced due to the absence of massive gauge boson decay channels. This leads to a large signal strength for the $\gamma Z$ and $\gamma \gamma$ channels. Therefore, current LHC data can exclude (or establish) this scenario.

Lastly we emphasize that our framework assumes 2HDM-II to be an effective low energy theory of a more complete theory. Our analysis suggests the 2HDM-II description of the electroweak symmetry breaking is only valid up to a scale around $1 \mathrm{TeV}$. Hence, if nature chooses to break electroweak symmetry by the 2HDM-II, there must be new particles waiting to be discovered with masses around $1 \mathrm{TeV}$.

\section{Acknowledgements}

In the final stages of this work Ref. [49-51] appeared. Our work is complementary to those work. We thank Jure Zupan for a critical reading of the manuscript. PU thanks the hospitality of theCERN Theory Division where part of this work is being completed. The work of BG is supported by the U.S. Department of Energy under contract No. DOE-FG03-97ER40546. PU is supported by DOE grant FG02-84-ER40153.

\section{A Loop-induced Decays of Neutral Scalar Bosons}

The expressions for loop-induced decays of the Standard Model Higgs boson, as well as for the MSSM neutral bosons decays, are given in Ref. [42, 52]. Here we give the rescaling factors for the partial decay width suitable for the 2HDM.

The rescaling factors for $\phi \rightarrow g g, \gamma \gamma$ where $\phi=h, H$ are

$$
\begin{aligned}
\frac{\Gamma_{g g}}{\Gamma_{g g}^{S M}} & =\left|\frac{\sum_{f} c_{t}^{\phi} A_{1 / 2}\left(\tau_{f}\right)}{\sum_{f} A_{1 / 2}\left(\tau_{f}\right)}\right|^{2}, \\
\frac{\Gamma_{\gamma \gamma}}{\Gamma_{\gamma \gamma}^{S M}} & =\left|\frac{\sum_{f} N_{C} Q_{f}^{2} c_{f}^{\phi} A_{1 / 2}\left(\tau_{f}\right)-c_{V}^{\phi} A_{1}\left(\tau_{W}\right)+\lambda_{\phi H^{+} H^{-}} A_{0}\left(\tau_{H^{ \pm}}\right)}{\sum_{f} N_{C} Q_{f}^{2} A_{1 / 2}\left(\tau_{f}\right)-A_{1}\left(\tau_{W}\right)}\right|^{2},
\end{aligned}
$$

where $N_{C}$ and $Q_{f}$ are the number of colors and the electric charge of fermion $f . \lambda_{\phi H^{+} H^{-}}$ is the trilinear coupling of $\phi$ to the charged Higgs boson in units of $2 M_{\phi}^{2} / v$. The 
functions $A_{i}$ give the 1-loop contribution of a spin- $i$ particle. They are given by

$$
\begin{aligned}
A_{0}(\tau) & =-\frac{1}{\tau^{2}}(\tau-f(\tau)), \\
A_{1 / 2}(\tau) & =\frac{2}{\tau^{2}}(\tau+(\tau-1) f(\tau)), \\
A_{1}(\tau) & =\frac{1}{\tau^{2}}\left(2 \tau^{2}+3 \tau+3(2 \tau-1) f(\tau)\right),
\end{aligned}
$$

and the function $f(\tau)$ is defined as

$$
f(\tau)= \begin{cases}\arcsin ^{2} \sqrt{\tau} & \tau \leq 1 \\ -\frac{1}{4}\left(\log \frac{1+\sqrt{1-1 / \tau}}{1-\sqrt{1-1 / \tau}}-i \pi\right)^{2} & \tau>1\end{cases}
$$

with $\tau_{i}=M_{\phi}^{2} / 4 M_{i}^{2}$ for $i=f, W$ and $H^{ \pm}$.

The rescaling factor for $\phi \rightarrow \gamma Z$ where $\phi=h, H$ is

$\frac{\Gamma_{\gamma Z}}{\Gamma_{\gamma Z}^{S M}}=\left|\frac{\sum_{f} N_{C} \frac{Q_{f}\left(2 I_{f}^{3}-4 Q_{f} s_{W}^{2}\right)}{c_{W}} c_{t}^{\phi} A_{1 / 2}^{\phi}\left(\tau_{f}, \lambda_{f}\right)+c_{V}^{\phi} A_{1}^{\phi}\left(\tau_{W}, \lambda_{W}\right)+\frac{c_{W}^{2}-s_{W}^{2}}{c_{W}} \lambda_{\phi H^{+} H^{-}} A_{0}^{\phi}\left(\tau_{H^{ \pm}}, \lambda_{H^{ \pm}}\right)}{\sum_{f} N_{C} \frac{Q_{f}\left(2 I_{f}^{3}-4 Q_{f} s_{W}^{2}\right)}{c_{W}} A_{1 / 2}^{\phi}\left(\tau_{f}, \lambda_{f}\right)+A_{1}^{\phi}\left(\tau_{W}, \lambda_{W}\right)}\right|^{2}$,

where $s_{W}\left(c_{W}\right)$ is the sine (cosine) of the weak mixing angle, $I_{f}^{3}$ is the left-hand weak isospin of the fermion $f, \tau_{i}=4 M_{i}^{2} / M_{\phi}^{2}$ and $\lambda_{i}=4 M_{i}^{2} / M_{Z}^{2}$. The function $A_{i}$ 's are defined as

$$
\begin{aligned}
A_{0}^{\phi}(\tau, \lambda) & =I_{1}(\tau, \lambda), \\
A_{1 / 2}^{\phi}(\tau, \lambda) & =I_{1}(\tau, \lambda)-I_{2}(\tau, \lambda), \\
A_{1}^{\phi}(\tau, \lambda) & =c_{W}\left\{4\left(3-\frac{s_{W}^{2}}{c_{W}^{2}}\right) I_{2}(\tau, \lambda)+\left[\left(1+\frac{2}{\tau}\right) \frac{s_{W}^{2}}{c_{W}^{2}}-\left(5+\frac{2}{\tau}\right)\right] I_{1}(\tau, \lambda)\right\},
\end{aligned}
$$

where the function $I_{1}$ and $I_{2}$ are defined as

$$
\begin{aligned}
& I_{1}(\tau, \lambda)=\frac{\tau \lambda}{2(\tau-\lambda)}+\frac{\tau^{2} \lambda^{2}}{2(\tau-\lambda)^{2}}\left(f\left(\tau^{-1}\right)-f\left(\lambda^{-1}\right)\right)+\frac{\tau^{2} \lambda}{(\tau-\lambda)^{2}}\left(g\left(\tau^{-1}\right)-g\left(\lambda^{-1}\right)\right), \\
& I_{2}(\tau, \lambda)=-\frac{\tau \lambda}{2(\tau-\lambda)^{2}}\left(f\left(\tau^{-1}\right)-f\left(\lambda^{-1}\right)\right) .
\end{aligned}
$$

The function $f$ is the same as in the $\phi \rightarrow \gamma \gamma$ case. The function $g$ is defined as

$$
g(\tau)= \begin{cases}\sqrt{\tau^{-1}-1} \arcsin \sqrt{\tau} & \tau \geq 1 \\ \frac{\sqrt{1-1 / \tau}}{2}\left(\log \frac{1+\sqrt{1-1 / \tau}}{1-\sqrt{1-1 / \tau}}-i \pi\right) & \tau<1\end{cases}
$$


For the CP-odd boson, its loop induced partial decay width into $g g, \gamma \gamma$ and $\gamma Z$ are given by [42]

$$
\begin{aligned}
\Gamma(A \rightarrow g g) & \simeq \frac{G_{F} \alpha_{s}^{2} M_{A}^{2}}{36 \sqrt{2} \pi^{3}}\left|\frac{3}{4} \cot \beta \frac{8 M_{t}^{2}}{M_{A}^{2}} f\left(M_{A}^{2} / 4 M_{t}^{2}\right)\right|^{2} \\
\Gamma(A \rightarrow \gamma \gamma) & \simeq \frac{G_{F} \alpha_{e m}^{2} M_{A}^{2}}{128 \sqrt{2} \pi^{3}}\left|\frac{4}{3} \cot \beta \frac{8 M_{t}^{2}}{M_{A}^{2}} f\left(M_{A}^{2} / 4 M_{t}^{2}\right)\right|^{2}, \\
\Gamma(A \rightarrow \gamma Z) & \simeq \frac{G_{F}^{2} M_{W}^{2} \alpha_{e m} M_{A}^{3}}{16 \pi^{4}}\left(1-\frac{M_{Z}^{2}}{M_{A}^{2}}\right)^{3}\left|2 \cot \beta \frac{1-\frac{8}{3} s_{W}^{2}}{c_{W}} \frac{8 M_{t}^{2}}{M_{A}^{2}} f\left(M_{A}^{2} / 4 M_{t}^{2}\right)\right|^{2}
\end{aligned}
$$

\section{B Beta-functions}

Here we list the one-loop beta-function for the gauge, Yukawa and scalar couplings. For the Yukawa couplings, we consider only the third generation contributions. We use the shorthand notation $\alpha_{i}=g_{i}^{2} /(4 \pi), \alpha_{y}=y^{2} /(4 \pi)$ and $\alpha_{\lambda}=\lambda /(4 \pi)$. The gauge coupling beta-functions are

$$
\beta_{\alpha_{1}}=\frac{7 \alpha_{1}^{2}}{2 \pi}, \quad \beta_{\alpha_{2}}=-\frac{3 \alpha_{2}^{2}}{2 \pi}, \quad \beta_{\alpha_{3}}=-\frac{7 \alpha_{3}^{2}}{2 \pi} .
$$

The Yukawa beta-functions are

$$
\begin{aligned}
\beta_{\alpha_{y t}} & =\frac{\alpha_{y_{t}}}{2 \pi}\left(\frac{9}{2} \alpha_{y_{t}}-\frac{3}{2} \alpha_{y_{b}}-\frac{17}{2} \alpha_{1}-\frac{9}{4} \alpha_{2}-8 \alpha_{3}\right) \\
\beta_{\alpha_{y b}} & =\frac{\alpha_{y_{b}}}{2 \pi}\left(\frac{9}{2} \alpha_{y_{b}}-\frac{3}{2} \alpha_{y_{t}}-\frac{17}{2} \alpha_{1}-\frac{9}{4} \alpha_{2}-8 \alpha_{3}\right) .
\end{aligned}
$$


Finally the scalar couplings beta-functions are

$$
\begin{aligned}
& \beta_{\alpha_{\lambda 1}}=\frac{1}{4 \pi}\left(12 \alpha_{\lambda_{1}}^{2}+4 \alpha_{\lambda_{3}}^{2}+4 \alpha_{\lambda_{3}} \alpha_{\lambda_{4}}+2 \alpha_{\lambda_{4}}^{2}+2 \alpha_{\lambda_{5}}^{2}\right. \\
& \left.+\frac{3}{4}\left(\alpha_{1}^{2}+3 \alpha_{2}^{2}+2 \alpha_{1} \alpha_{2}\right)-3 \alpha_{\lambda_{1}}\left(\alpha_{1}+3 \alpha_{2}-4 \alpha_{y_{b}}\right)-12 \alpha_{y_{b}}^{2}\right) \\
& \beta_{\alpha_{\lambda 2}}=\frac{1}{4 \pi}\left(12 \alpha_{\lambda_{2}}^{2}+4 \alpha_{\lambda_{3}}^{2}+4 \alpha_{\lambda_{3}} \alpha_{\lambda_{4}}+2 \alpha_{\lambda_{4}}^{2}+2 \alpha_{\lambda_{5}}^{2}\right. \\
& \left.+\frac{3}{4}\left(\alpha_{1}^{2}+3 \alpha_{2}^{2}+2 \alpha_{1} \alpha_{2}\right)-3 \alpha_{\lambda_{2}}\left(\alpha_{1}+3 \alpha_{2}-4 \alpha_{y_{b}}\right)-12 \alpha_{y_{b}}^{2}\right) \\
& \beta_{\alpha_{\lambda 3}}=\frac{1}{4 \pi}\left(2\left(\alpha_{\lambda_{1}}+\alpha_{\lambda_{2}}\right)\left(3 \alpha_{\lambda_{3}}+\alpha_{\lambda_{4}}\right)+4 \alpha_{\lambda_{3}}^{2}+2 \alpha_{\lambda_{4}}^{2}+2 \alpha_{\lambda_{5}}^{2}\right. \\
& \left.+\frac{3}{4}\left(\alpha_{1}^{2}+3 \alpha_{2}^{2}+2 \alpha_{1} \alpha_{2}\right)-3 \alpha_{\lambda_{3}}\left(\alpha_{1}+3 \alpha_{2}-2 \alpha_{y_{t}}-2 \alpha_{y_{b}}\right)\right) \\
& \beta_{\alpha_{\lambda 4}}=\frac{1}{4 \pi}\left(2\left(\alpha_{\lambda_{1}}+\alpha_{\lambda_{2}}\right) \alpha_{\lambda_{4}}+8 \alpha_{\lambda_{3}} \alpha_{\lambda_{4}}+4 \alpha_{\lambda_{4}}^{2}+8 \alpha_{\lambda_{5}}^{2}\right. \\
& \left.+3 \alpha_{1} \alpha_{2}-3 \alpha_{\lambda_{4}}\left(\alpha_{1}+3 \alpha_{2}-2 \alpha_{y_{t}}-2 \alpha_{y_{b}}\right)\right) \\
& \beta_{\alpha_{\lambda 5}}=\frac{1}{4 \pi}\left(2\left(\alpha_{\lambda_{1}}+\alpha_{\lambda_{2}}\right) \alpha_{\lambda_{5}}+8 \alpha_{\lambda_{3}} \alpha_{\lambda_{5}}+12 \alpha_{\lambda_{4}} \alpha_{\lambda_{5}}\right. \\
& \left.-3 \alpha_{\lambda_{5}}\left(\alpha_{1}+3 \alpha_{2}-2 \alpha_{y_{t}}-2 \alpha_{y_{b}}\right)\right)
\end{aligned}
$$

\section{References}

[1] ATLAS Collaboration Collaboration, G. Aad et al., Observation of a new particle in the search for the Standard Model Higgs boson with the ATLAS detector at the LHC, Phys.Lett. B716 (2012) 1-29, [arXiv:1207.7214].

[2] CMS Collaboration Collaboration, S. Chatrchyan et al., Observation of a new boson at a mass of $125 \mathrm{GeV}$ with the CMS experiment at the LHC, Phys.Lett. B716 (2012) 30-61, [arXiv:1207.7235].

[3] CDF Collaboration, Do Collaboration Collaboration, T. Aaltonen et al., Evidence for a particle produced in association with weak bosons and decaying to a bottom-antibottom quark pair in Higgs boson searches at the Tevatron, Phys.Rev.Lett. 109 (2012) 071804, [arXiv:1207.6436].

[4] D. Carmi, A. Falkowski, E. Kuflik, T. Volansky, and J. Zupan, Higgs After the Discovery: A Status Report, arXiv:1207.1718. 
[5] J. Espinosa, C. Grojean, M. Muhlleitner, and M. Trott, First Glimpses at Higgs' face, arXiv:1207.1717.

[6] T. Corbett, O. Eboli, J. Gonzalez-Fraile, and M. Gonzalez-Garcia, Robust Determination of the Higgs Couplings: Power to the Data, Phys.Rev. D87 (2013) 015022, [arXiv: 1211.4580].

[7] A. Azatov and J. Galloway, Electroweak Symmetry Breaking and the Higgs Boson: Confronting Theories at Colliders, Int.J.Mod.Phys. A28 (2013) 1330004, [arXiv: 1212.1380].

[8] Observation of an excess of events in the search for the standard model higgs boson in the gamma-gamma channel with the atlas detector, Tech. Rep.

ATLAS-CONF-2012-091, CERN, Geneva, Jul, 2012.

[9] Observation of an excess of events in the search for the Standard Model Higgs boson in the $H \rightarrow Z Z^{(*)} \rightarrow 4 \ell$ channel with the ATLAS detector., Tech. Rep.

ATLAS-CONF-2012-092, CERN, Geneva, Jul, 2012.

[10] Update of the $\boldsymbol{H} \rightarrow \boldsymbol{W} \boldsymbol{W}^{(*)} \rightarrow \boldsymbol{e} \boldsymbol{\nu} \boldsymbol{\mu \nu}$ analysis with $13 \mathrm{fb}^{-1}$ of $\sqrt{s}=8$ tev data collected with the atlas detector, Tech. Rep. ATLAS-CONF-2012-158, CERN, Geneva, Nov, 2012.

[11] Combination of standard model higgs boson searches and measurements of the properties of the new boson with a mass near 125 gev, Tech. Rep.

CMS-PAS-HIG-12-045, CERN, Geneva, Nov, 2012.

[12] TEVNPH (Tevatron New Phenomina and Higgs Working Group), CDF Collaboration, D0 Collaboration Collaboration, Combined CDF and DO Search for Standard Model Higgs Boson Production with up to $10.0 \mathrm{fb}^{-1}$ of Data, arXiv: 1203.3774.

[13] Measurements of the properties of the higgs-like boson in the four lepton decay channel with the atlas detector using $25 \mathrm{fb}$ ? 1 of proton-proton collision data, Tech. Rep.

ATLAS-CONF-2013-013, CERN, Geneva, Mar, 2013.

[14] Properties of the higgs-like boson in the decay $h$ to zz to $4 \mathrm{l}$ in pp collisions at sqrt $s=7$ and 8 tev, Tech. Rep. CMS-PAS-HIG-13-002, CERN, Geneva, 2013.

[15] Evidence for a particle decaying to $w+w$-in the fully leptonic final state in a standard model higgs boson search in pp collisions at the lhc, Tech. Rep. CMS-PAS-HIG-13-003, CERN, Geneva, 2013.

[16] G. Branco, P. Ferreira, L. Lavoura, M. Rebelo, M. Sher, et al., Theory and phenomenology of two-Higgs-doublet models, Phys.Rept. 516 (2012) 1-102, [arXiv:1106.0034]. 
[17] H. Cheon and S. K. Kang, Constraining parameter space in type-II two-Higgs doublet model in light of a 125 GeV Higgs boson, arXiv:1207.1083.

[18] Y. Bai, V. Barger, L. L. Everett, and G. Shaughnessy, The 2HDM-X and Large Hadron Collider Data, arXiv:1210.4922.

[19] W. Altmannshofer, S. Gori, and G. D. Kribs, A Minimal Flavor Violating 2HDM at the $L H C$, arXiv:1210.2465.

[20] A. Drozd, B. Grzadkowski, J. F. Gunion, and Y. Jiang, Two-Higgs-Doublet Models and Enhanced Rates for a 125 GeV Higgs, arXiv:1211.3580.

[21] J. Chang, K. Cheung, P.-Y. Tseng, and T.-C. Yuan, Implications on the Heavy CP-even Higgs Boson from Current Higgs Data, arXiv: 1211.3849.

[22] C.-Y. Chen and S. Dawson, Exploring Two Higgs Doublet Models Through Higgs Production, arXiv:1301.0309.

[23] A. Goudelis, B. Herrmann, and O. Stl, Dark matter in the Inert Doublet Model after the discovery of a Higgs-like boson at the LHC, arXiv:1303.3010.

[24] P. Ferreira and D. Jones, Bounds on scalar masses in two Higgs doublet models, JHEP 0908 (2009) 069, [arXiv:0903.2856].

[25] S. L. Glashow and S. Weinberg, Natural Conservation Laws for Neutral Currents, Phys.Rev. D15 (1977) 1958.

[26] R. Peccei and H. R. Quinn, Constraints Imposed by CP Conservation in the Presence of Instantons, Phys.Rev. D16 (1977) 1791-1797.

[27] S. Weinberg, A New Light Boson?, Phys.Rev.Lett. 40 (1978) 223-226.

[28] F. Wilczek, Problem of Strong $p$ and $t$ Invariance in the Presence of Instantons, Phys.Rev.Lett. 40 (1978) 279-282.

[29] P. P. Giardino, K. Kannike, M. Raidal, and A. Strumia, Reconstructing Higgs boson properties from the LHC and Tevatron data, JHEP 1206 (2012) 117, [arXiv: 1203.4254].

[30] A. Azatov, R. Contino, and J. Galloway, Model-Independent Bounds on a Light Higgs, JHEP 1204 (2012) 127, [arXiv:1202.3415].

[31] ATLAS Collaboration Collaboration, G. Aad et al., Search for the Standard Model Higgs boson in the diphoton decay channel with $4.9 \mathrm{fb}-1$ of pp collisions at sqrt(s)=7 TeV with ATLAS, Phys.Rev.Lett. 108 (2012) 111803, [arXiv:1202.1414].

[32] Evidence for a new state decaying into two photons in the search for the standard model higgs boson in pp collisions, Tech. Rep. CMS-PAS-HIG-12-015, CERN, Geneva, Jul, 2012. 
[33] ATLAS Collaboration Collaboration, G. Aad et al., Search for the Standard Model Higgs boson in the decay channel $H \rightarrow Z Z^{(*)} \rightarrow 4 \ell$ with $4.8 \mathrm{fb}-1$ of pp collision data at sqrt(s) = 7 TeV with ATLAS, Phys.Lett. B710 (2012) 383-402, [arXiv:1202.1415].

[34] Combination of SM, SM4, FP Higgs boson searches, Tech. Rep. CMS-PAS-HIG-12-008, CERN, Geneva, Feb, 2012.

[35] Search for the Standard Model Higgs boson in the $H \rightarrow W W^{(*)} \rightarrow \ell \nu \ell \nu$ decay mode with $4.7 \mathrm{fb}-1$ of ATLAS data at sqrt(s) = 7 TeV, Tech. Rep. ATLAS-CONF-2012-012, CERN, Geneva, Mar, 2012.

[36] Search for the standard model higgs boson in $h \rightarrow$ tau tau decays in proton-proton collisions with the atlas detector, Tech. Rep. ATLAS-CONF-2012-160, CERN, Geneva, Nov, 2012.

[37] LHC Higgs Cross Section Working Group, S. Dittmaier, C. Mariotti, G. Passarino, and R. Tanaka (Eds.), Handbook of LHC Higgs Cross Sections: 1. Inclusive Observables, CERN-2011-002 (CERN, Geneva, 2011) [arXiv:1101.0593].

[38] ALEPH Collaboration, DELPHI Collaboration, L3 Collaboration, OPAL Collaboration, The LEP working group for Higgs boson searches Collaboration, G. Abbiendi et al., Search for Charged Higgs bosons: Combined Results Using LEP Data, arXiv:1301.6065.

[39] T. Hermann, M. Misiak, and M. Steinhauser, $\bar{B} \rightarrow X_{s} \gamma$ in the Two Higgs Doublet Model up to Next-to-Next-to-Leading Order in QCD, JHEP 1211 (2012) 036, [arXiv: 1208.2788].

[40] ALEPH Collaboration, DELPHI Collaboration, L3 Collaboration, OPAL Collaboration, LEP Working Group for Higgs Boson Searches Collaboration, S. Schael et al., Search for neutral MSSM Higgs bosons at LEP, Eur.Phys.J. C47 (2006) 547-587, [hep-ex/0602042].

[41] M. Baak, M. Goebel, J. Haller, A. Hoecker, D. Kennedy, et al., The Electroweak Fit of the Standard Model after the Discovery of a New Boson at the LHC, arXiv:1209.2716.

[42] A. Djouadi, The Anatomy of electro-weak symmetry breaking. II. The Higgs bosons in the minimal supersymmetric model, Phys.Rept. 459 (2008) 1-241, [hep-ph/0503173].

[43] Search for the standard model higgs boson in the $h \rightarrow z \gamma$ decay mode with pp collisions at $\sqrt{s}=7$ and 8 tev, Tech. Rep. ATLAS-CONF-2013-009, CERN, Geneva, Mar, 2013.

[44] Measurements of the properties of the higgs-like boson in the two photon decay channel with the atlas detector using $25 \mathrm{fb}^{-1}$ of proton-proton collision data, Tech. Rep. ATLAS-CONF-2013-012, CERN, Geneva, Mar, 2013.

[45] Search for the standard model higgs boson in the z boson plus a photon channel in pp 
collisions at sqrt-s $=7$ and 8 tev, Tech. Rep. CMS-PAS-HIG-13-006, CERN, Geneva, 2013.

[46] Updated measurements of the higgs boson at 125 gev in the two photon decay channel, Tech. Rep. CMS-PAS-HIG-13-001, CERN, Geneva, 2013.

[47] J. F. Gunion, H. E. Haber, G. L. Kane, and S. Dawson, THE HIGGS HUNTER'S GUIDE, Front.Phys. 80 (2000) 1-448.

[48] F. Borzumati, J.-L. Kneur, and N. Polonsky, Higgs-Strahlung and R-parity violating slepton-Strahlung at hadron colliders, Phys.Rev. D60 (1999) 115011, [hep-ph/9905443].

[49] A. Celis, V. Ilisie, and A. Pich, LHC constraints on two-Higgs doublet models, arXiv: 1302.4022.

[50] C.-W. Chiang and K. Yagyu, Implications of Higgs boson search data on the two-Higgs doublet models with a softly broken $Z_{2}$ symmetry, arXiv:1303.0168.

[51] A. Barroso, P. Ferreira, I. Ivanov, and R. Santos, Metastability bounds on the two Higgs doublet model, arXiv:1303.5098.

[52] A. Djouadi, The Anatomy of electro-weak symmetry breaking. I: The Higgs boson in the standard model, Phys.Rept. 457 (2008) 1-216, [hep-ph/0503172]. 